$\begin{array}{ll} & \text { solution } \\ \mathrm{H} & =\text { hydrogen ion } \\ \mathrm{HCl} & =\text { hydrochloric acid } \\ \mathrm{H}_{2} \mathrm{~L} & =\text { active oxime in LIX65N (free oxime) } \\ 0 & =\text { interface between membrane and feed solution }\end{array}$

Literature Cited

1) Ashbrook, A. W.: Coord. Chem. Rev., 16, 285 (1975).

2) idem: J. Chromatograph, 105, 141 (1975).

3) Baker, R.W., M.E. Tuttle, D.J. Kelly and H.K. Lonsdale: J. Membrane Sci., 2, 213 (1977).

4) Choy, E. M., D. F. Evans and E. L. Cussler: J. Am. Chem. Soc., 96, 7085 (1974).

5) Crank, J.: "The Mathematics of Diffusion". p. 49, Oxford Univ. Press (1975).

6) Cussler, E. L.: $A I C h E J ., 17,1300$ (1971).

7) Duffey, M. E., D. F. Evans and E. L. Cussler: J. Membrane Sci., 3, 1 (1978).

8) Hochhauser, A. M. and E. L. Cussler: AIChE J. Symp. Ser., 71, 136 (1975).

9) Hughes, M. A., H. C. Sergeant, R. J. Whewell: J. Inorg.
Nucl. Chem., 41, 1603 (1979).

10) Kataoka, T., T. Nishiki, Y. Tamura and K. Ueyama: $J$. Chem. Eng. Japan, 13, 35 (1980).

11) Kojima, T., J. Tomita and T. Miyauchi: Kagaku Kogaku Ronbunshu, 5, 476 (1979)

12) Komasawa, I., T. Otake and A. Yamada: J. Chem. Eng. Japan, 13, 130 (1980).

13) Largman, T. and S. Sifniades: Hydrometallurgy 3, 153 (1978).

14) Lee, K. H., D. F. Evans and E. L. Cussler: $A I C h E J ., 24$, 860 (1978).

15) Mackie, J. S. and P. Meares: Proc. Roy. Soc. London, Ser. A, 232, 498 (1955).

16) Martin, T. P. and G. A. Davies: Hydrometallurgy, 2, 315 (1976/1977).

17) Völkel, W., W. Halwachs and K. Schügerl: J. Membrane Sci., 6, 19 (1980).

18) Ward III, E. J.: AIChE J., 16, 405 (1970).

19) Whewell, R. J., M. A. Hughes and C. Hansen: J. Inorg. Nucl. Chem., 38, 2071 (1976).

\title{
COMPOSITION OF SOLID CONDENSATE ON A COLD SURFACE FROM A MIXED ORGANIC VAPOR
}

\author{
Masakuni MATSUOKA and Hidekazu KOMIYA \\ Department of Chemical Engineering, \\ Tokyo University of Agriculture and Technology, Tokyo 184
}

\begin{abstract}
A method to estimate the composition of solid condensate from a binary vapor in fractional sublimation accompanied by an entrainer gas is derived, including an overall coefficient for condensation of the individual components. The coefficient was empirically correlated with gas flow rates, surface temperatures of condenser, and condenser dimensions. Good agreement between the estimated and observed compositions of the condensates was obtained for two eutectic-forming organic mixtures and one solid solution organic mixture.
\end{abstract}

\section{Introduction}

Although sublimation has been used to purify a number of substances in the laboratory and in industry its practical use has been limited to the removal and/or recovery of one or more volatile components from a solid mixture ${ }^{3)}$. In the case of one volatile component, the process is essentially condensation of a single vapor from a gas. Meanwhile, condensation of a mixed vapor composed of volatile substances is considered to form a solid mixture of which compositions are determined by the operating conditions

\footnotetext{
Received October 4, 1980. Correspondence concerning this article should be addressed to M. Matsuoka. H. Komiya is at IBM Japan, Ltd., Tokyo 106.
}

as well as the phase equilibria. Thus more knowledge of the factors affecting the rate of condensation and resulting solids physical properties is required ${ }^{4}$. However, studies of fractional sublimation ${ }^{3,6,7)}$ have been aimed mainly at the development of apparatus, and methods of estimation of product compositions have not been established.

Among the studies of fractional sublimation, Reid ${ }^{3}$, who seems to be the first author in this field, presented the idea of fractionation by utilizing the solid-vapor phase change in a continuous countercurrent multistage apparatus. He carried out several runs at a reduced pressure with a helical tube of six turns rotating on a horizontal axis, which is partially sub- 
merged in a coolant with vaporizing heat applied to the upper part. Since the temperatures given at the vaporizing and condensing sections deviated greatly from the equilibrium values, an analogy with fractional distillation is questionable, contrary to his intention. Nevertheless, he reported the feasibility of the separation of benzene and cyclohexane mixtures.

Gillot and Goldberger ${ }^{3)}$ constructed a countercurrent apparatus also based on the analogy with fractional distillation in which a condensed layer forms a thin film on a descending bed of solid particles while the vapor is carried upward by an entrainer gas to facilitate equilibration between the phases. With this apparatus they obtained 4.2 theoretical stages for the separation of $p$-dibromobenzene and $p$-bromochlorobenzene mixtures in a column $34 \mathrm{~cm}$ in height at total reflux.

A recent work by Vitovec et al. ${ }^{7)}$ described a continuous fractional sublimation column in which condensation occurs by evaporation cooling of the dispersed water, and the results were reported in terms of the yield and composition of the product for naphthalene and phthalic anhydride mixtures. However, the condensation conditions were not specified, and there was no discussion of the relations between composition and operating conditions.

The purpose of the present study is to provide a method of estimating the composition of solid organic condensates from a vapor-gas mixture on a cold surface of a simple double-tube condenser.

\section{Theory}

Consider a case in which an unsaturated binary organic vapor mixture in an entrainer gas is brought into contact with a cold surface of a double-tube condenser. Since vapor pressures of most organic solids which are considered for fractional sublimation are of the order of $1 \mathrm{mmHg}$, rates of mass transfer of the individual components in the entrainer gas can be assumed to be independent of each other. Furthermore, if one assumes that the rate of condensation of component $i$ from the vapor-gas mixture is proportional to the apparent supersaturation at the interface, one can write the rate of condensation as

$$
d N_{i}=K_{i} C\left(y_{i}-y_{i}^{*}\right) d A
$$

where $K_{i}$ is a local overall coefficient for condensation, $y_{i}^{*}$ is an equilibrium composition at the interface temperature $\left(t_{s}\right)$ in terms of mole fraction and $d A$ denotes the infinitesimal surface area along the tube $(=\pi D d l)$. The composition of the solid condensate at the location can be written as

$$
z_{A}=d N_{A} /\left(d N_{A}+d N_{B}\right)
$$

where subscripts $A$ and $B$ denote the components of the mixture. Equation (1) can be integrated to give total condensation rates after being combined with the following mass balance equation:

$$
d N_{i}=-\bar{u} S C d y_{i}
$$

Hence the total condensation rate $N_{i}$ can be expressed in terms of the inlet supersaturation as

$$
N_{i}=k_{i} A C\left(y_{i 1}-y_{i}^{*}\right)
$$

with the definition of

$$
\begin{aligned}
k_{i} & \equiv(D \bar{u} / 4 L)\left[1-\exp \left(-4 L \bar{K}_{i} / D \bar{u}\right)\right] \\
\text { and } \quad \bar{K}_{i} & \equiv \int_{0}^{L} K_{i} d l / L
\end{aligned}
$$

where $y^{*}$ is assumed to be constant (Appendix). The average product composition $Z_{A}$ is then expressed as

$$
Z_{A}=N_{A} /\left(N_{A}+N_{B}\right)
$$

Substituting the individual rates of condensation in Eq. (7) one obtains the following equation for $Z_{A}$ :

$$
Z_{A}=\frac{\alpha\left(y_{A 1}-y_{A}^{*}\right)}{\alpha\left(y_{A 1}-y_{A}^{*}\right)+\left(y_{B 1}-y_{B}^{*}\right)}
$$

where

$$
\alpha=\frac{k_{A}}{k_{B}}=\frac{1-\exp \left(-4 L \bar{K}_{A} / D \bar{u}\right)}{1-\exp \left(-4 L \bar{K}_{B} / D \bar{u}\right)}
$$

Now the composition of solid condensates can be estimated from Eq. (8), if one knows the values of $\alpha$ and the inlet and equilibrium compositions, where $\alpha$ is dependent on $\bar{K}_{i}$ and on other variables in Eq. (9). Since $\bar{K}_{i}$ is considered to include the coefficients for mass transfer in the gas, heat transfer via surface temperatures, surface kinetics and probably nucleation processes, it should be a complicated function of the gas flow characteristics, thermal conditions, condenser dimensions and probably conditions of the cold surface. Therefore it is necessary to know the relations between $\bar{K}_{i}$ and these variables beforehand, experimentally or by some other adequate method, in order to estimate the composition of solid condensates.

\section{Experimental Apparatus and Procedure}

The schematic diagram of the appararus used in the experiments on condensation of binary mixtures is shown in Fig. 1. A flow of nitrogen gas as an entrainer was divided into the two streams which were led to separate baths, different in temperatures, after each flow rate was controlled. A set of a copper coil ( $6 \mathrm{~mm}$ in diameter and $1 \mathrm{~m}$ in length) and a glass saturater (19 $\mathrm{mm}$ in diameter and $250 \mathrm{~mm}$ in length) was placed in each bath, through which the gas was pre-heated and saturated with the vapor of the organic solid at the bath temperature. After being mixed in the higher-temperature bath, the gas was brought into contact with a cold surface of a condenser placed in the same bath. The condenser consisted of a copper tube $15 \mathrm{~mm}$ in I.D. and $85 \mathrm{~mm}$ in length sandwiched 
with glass tubes which acted as calming sections, each of $85 \mathrm{~mm}$ and $65 \mathrm{~mm}$ in length, and was jacketted with a glass tube. Through the annular space cooling water was allowed to flow at a known temperature and flow rate. The gas from the condenser was finally led to a total condenser to recover the rest of the organic vapors and then purged.

The temperatures in the apparatus were measured with copper-constantan thermocouples. The systems chosen were benzoic acid-naphthalene and naphthalene- $p$-dichlorobenzene eutectic-forming mixtures, and a $p$-dibromobenzene- $p$-dichlorobenzene solid solution mixture.

Quantitative analysis of the condensate was made with a F.I.D. gas chromatograph with calibration curves, while the vapor composition in the gas was determined from the flow rates of the gas streams and the equilibrium vapor pressures at the bath temperatures.

Prior to the binary condensation expriments, the vapor pressure and the rates of condensation of the individual organics were measured. The vapor pressure was determined as follows: using a single gas stream system, the change in weight of the solid in the saturater before and after passage of the known amount of inert gas was measured. It was then converted to the vapor pressure with the assumption of the ideal gas law. The rate of condensation of the individual vapors was obtained in a similar manner as those for the binary experiments except that only a single gas stream was utilized. The weight of condensate on the surface was measured after being scraped off, then the rate of condensation in $\mathrm{mol} / \mathrm{sec}$ was calculated. In addition, the condensation rate was also measured in condensers $21 \mathrm{~mm}$ and $42.5 \mathrm{~mm}$ in length to find the effects of tube length.

\section{Results and Discussion}

\section{1 Vapor pressures}

The measured vapor pressures of the four solids were plotted on semi-log paper as in Fig. 2, and were found to give straight lines over the measured temperature ranges. The resulting equations are:

for naphthalene

$$
\begin{array}{cc}
\quad \log p=11.314-3690 / T & 10<t<80^{\circ} \mathrm{C} \\
\text { for benzoic acid } & \\
\quad \log p=12.834-4762 / T & 30<t<100^{\circ} \mathrm{C} \\
\text { for } p-\mathrm{DCB} & \\
\quad \log p=11.373-3390 / T & 10<t<50^{\circ} \mathrm{C} \\
\text { and for } p \text {-DBB } & \\
\quad \log p=11.592-3826 / T & 10<t<80^{\circ} \mathrm{C}
\end{array}
$$

Since agreement with the literature data ${ }^{1,2,8\rangle}$ is very good for each solid, the gas at the outlet of the saturater is considered to be completely saturated with the vapor. The above equations are to be used in the

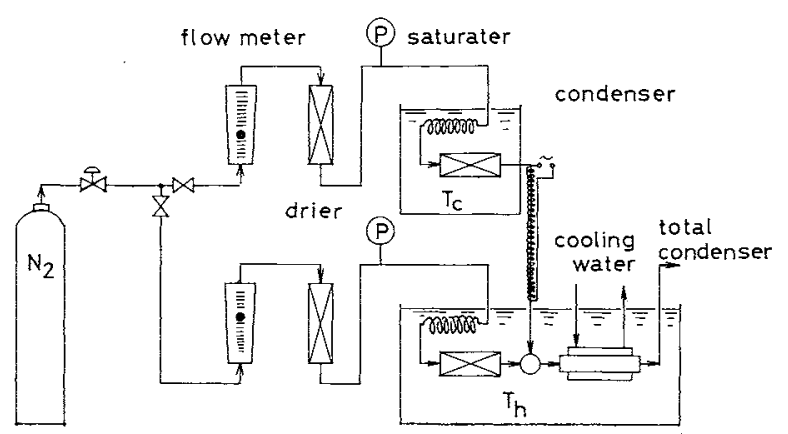

Fig. 1 Schematic diagram of experimental apparatus

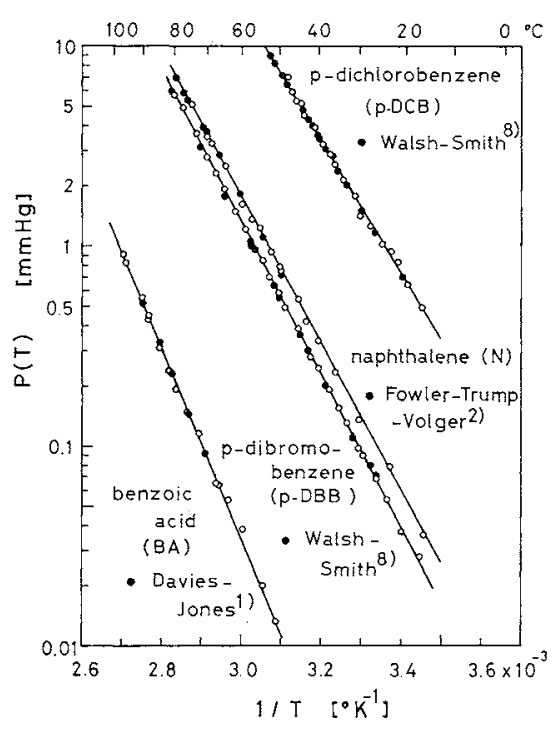

Fig. 2 Measured vapor pressures

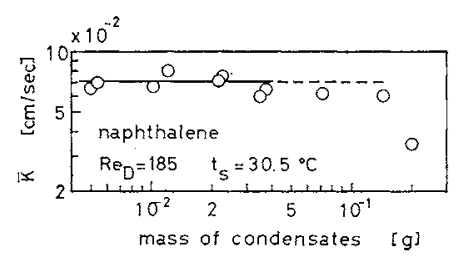

Fig. 3 Effect of amount of condensate on $\bar{K}_{i}$

following calculation.

\section{2 Condensation rates of single vapors}

\section{1) Effects of the amount of condensate}

The crystals condensed on the cold surface did not appear as a dense layer but as a good many tiny scales. Therefore, as the amount increases the interfacial area also increases, which can result in the increase of the apparent codensation rate. At the same time, the interface gradually protrudes into the hot main gas stream, which can on the contrary reduce the condensation rate. Thus the effects of the amount of condensates were experimentally investigated as shown in Fig. 3 for naphthalene, revealing that the rate gradually decreases so that the latter of the two mechanisms seems to affect more strongly the condensation rate. On the basis of this fact the amount 


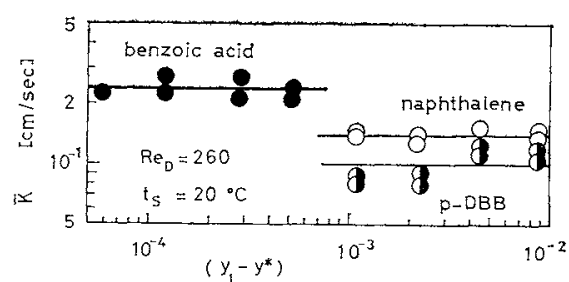

Fig. 4 Effect of inlet supersaturation on $\overline{K_{i}}$

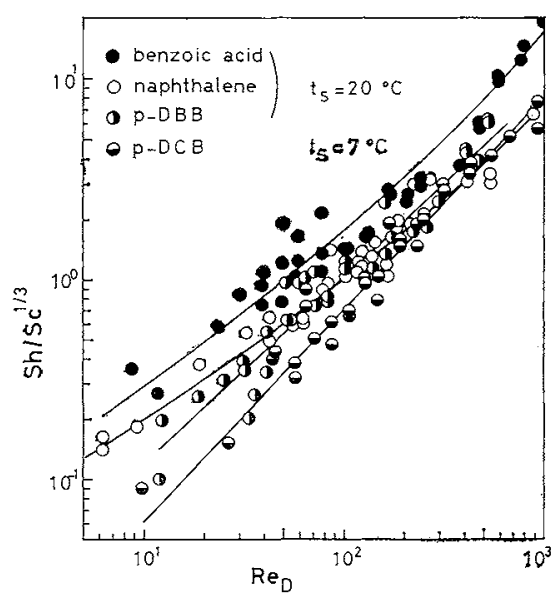

Fig. 5 Correlation of condensation rates at constant surface iemperature

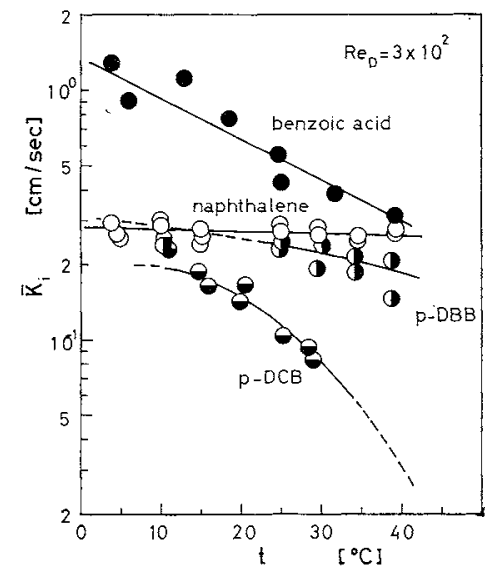

Fig. 6 Effect of surface temperature on $\bar{K}_{i}$

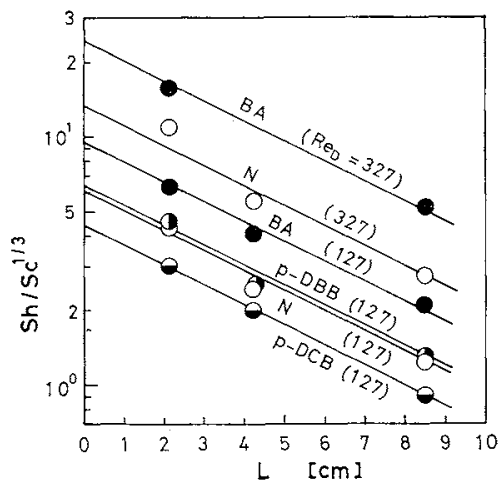

Fig. 7 Effect of tube length on $S h / S c^{1 / 3}$ was decided to be less than $0.03 \mathrm{~g}$ in the following experiments.

2) Flow rate dependence

An examination of Eq. (1) was made by varying the apparent supersaturation at the inlet. As seen from Fig. 4, $\bar{K}_{i}$ is almost independent of the driving force, thus the proportionality assumed in Eq. (1) was understood to hold. Though the data for $p$-DBB seem to show some dependence, it is considered that all of the four materials obey Eq. (1).

The condensation rates under the conditions of constant surface temperature and various gas velocities were correlated in terms of the dimensionless numbers $S h\left(=\bar{K}_{i} D / \mathscr{D}\right), S c$ and $\operatorname{Re}_{\mathrm{D}}(=\rho D \bar{u} / \mu)$, and are shown in Fig. 5, where the surface temperature was kept at about $20^{\circ} \mathrm{C}$ except for $p$-DCB, for which it was at $7^{\circ} \mathrm{C}$. Figure 5 shows that the condensation rate varies considerably with gas velocity and differs for different materials. Accordingly, the condensation rate is understood to be controlled by not only the mass transfer rate in the gas but also other rate processes such as surface kinetics.

\section{3) Dependence on surface temperatures}

The dependence of $\bar{K}_{i}$ on the surface temperature is shown in Fig. 6 under the conditions of constant gas flow rate and constant inlet composition for each system. What is common to the four organics is the tendency of decrease in value of $\bar{K}_{i}$ with increasing surface temperature. Though this is just the opposite of what is usually observed in crystallization from a solution or a melt, the curves in Fig. 6 are used to correct the values of $\bar{K}_{i}$ obtained at a particular temperature to those at any desired temperature. This fact also suggests that some surface kinetics participates the condensation process which is governed by the dimensionless driving force $\left(y_{i}-y_{i}^{*}\right) / y_{i}^{*}$.

4) Effect of condenser length

Similar experiments using condensers different in length were carried out. Figure 7 shows the relations of $\bar{K}_{i}$ and $L$ at constant Reynolds numbers. It was found that the same data can also be plotted on loglog paper to give a relation $\bar{K}_{i} \propto L^{-1}$. Because the surface rate process is believed to exist even at the inlet section, $\bar{K}_{i}$ cannot be infinite there. Thus it may be reasonable to take the former relation. As seen from Fig. 7, the solpes are found to be all equal. The fact that the values of $\bar{K}_{i}$ vary markedly with $L$ indicates that the condensation is much affected by a sudden change in temperature, hence in equilibrium vapor pressure, and this is a very important factor in designing a condenser for a fractional sublimation process. Further study of the condensation rate is necessary and will be presented elsewhere ${ }^{5}$.

\section{3 Condensation of binary mixtures}

The compositions of solid condensates for the three 


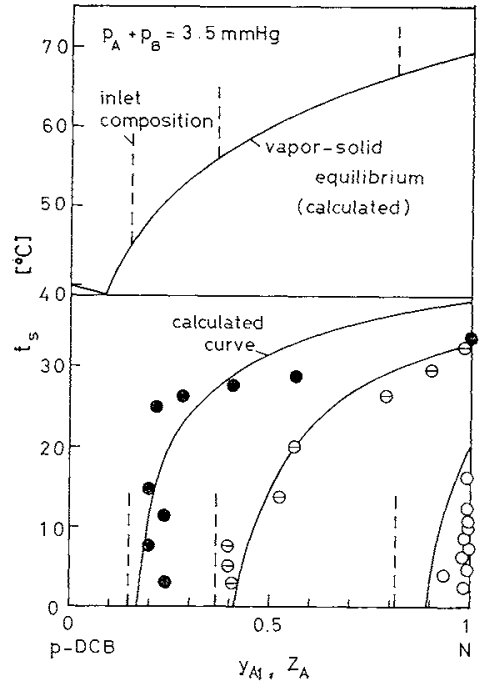

Fig. 8 Composition of solid condensate vs. surface temperature: for $p$-DCB-Naphthalene system

systems against the cooling surface temperatures are shown in Figs. 8, 9 and 10. The gas flow rates and the total vapor pressures were kept constant throughout the runs for each system. The composition, expressed in terms of mole fraction of the organics excluding the inert gas, is found to be higher for higher surface temperature and to approach the vapor composition itself for extremely lower temperatures.

The curves were drawn in accordance with Eq. (8), as will be described below.

\section{4 Estimation of compositions of solid condensates}

\section{1) Eutectic systems}

For simple eutectic systems, the partial vapor pressure of each component at the surface is assumed to be equal to its own vapor pressure at that temperature, hence

$$
y_{i}^{*}=P_{i}\left(T_{s}\right) / \pi \quad(i=A \text { and } B)
$$

Thus $Z_{A}$ can be calculated by substituting the values of $\alpha, y_{i}$ and $y_{i}^{*}$ in Eq. (8), knowing only the flow condition and the surface temperature. The solid curves in Figs. 8 and 9 show the calculated relations. Here the calculated curves approach unity below the eutectic temperatures because of the presence of the entrainer gas, which adds one more freedom in the phase rule. Good agreement was obtained for the system of naphthalene and $p$-DCB between the calculated and experimental values, but discrepancies were found for the benzoic acid-naphthalene system. This may be due to the fact that benzoic acid condensed as fine powder-like crystalline particles different from the other three substances, so that condensation of benzoic acid along the condenser surface occurred preferentially since the crystal particles of benzoic acid protrude less into the gas stream where the temperature is higher than at the interface.

2) Solid solution system

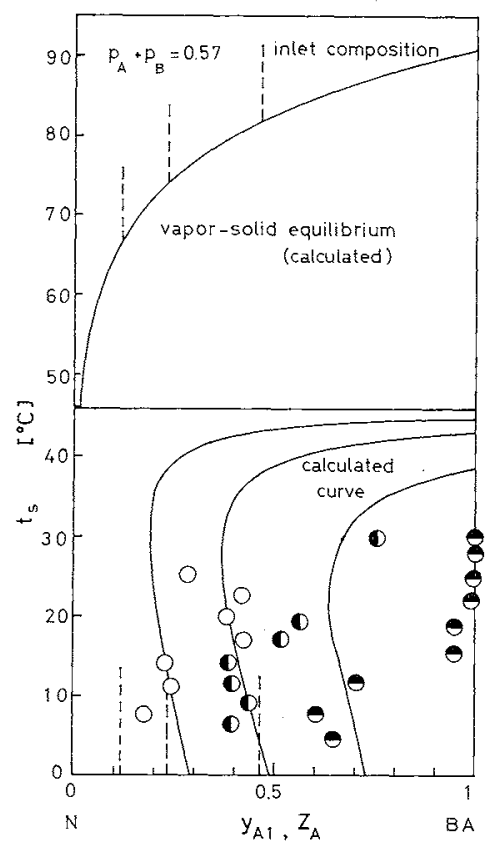

Fig. 9 Composition of solid condensate vs. surface temperature: for Benzoic acid-Naphthalene system

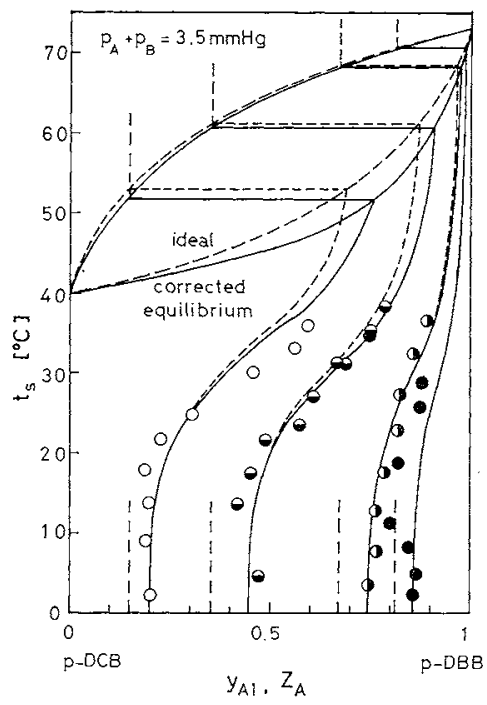

Fig. 10 Composition of solid condensate vs. surface temperature: for $p$-DBB-p-DCB system

Assuming Raoult's law to hold for the vapor-solid phase equilibrium and taking into account the nonideal behavior of the system, the equilibrium vapor composition at the interface can be given as

$$
y_{i}^{*}=\gamma_{i} P_{i}\left(T_{s}\right) Z_{i} / \pi \quad(i=A \text { and } B)
$$

Substituting these in Eq. (8) and applying algebraic treatment lead to the following equation for the composition of solid condensates.

$$
\begin{aligned}
Z_{A}= & (1 / 2 \beta)\left[-\left(\alpha y_{A 1}+y_{B 1}-\beta\right)\right. \\
& \left.+\sqrt{\left(\alpha y_{A 1}+y_{B 1}-\beta\right)^{2}+4 \alpha \beta y_{A 1}}\right]
\end{aligned}
$$

where

$$
\beta=\left[\gamma_{B} P_{B}\left(T_{s}\right)-\gamma_{A} P_{A}\left(T_{s}\right)\right] / \pi
$$


The solid curves in Fig. 10 represent the estimated temperature-composition relations using the values of $\gamma_{i}$ from ref. 9), with the assumption that the activity coefficients of the system are the same as those measured at a constant temperature.

The dotted curves in Fig. 10 are drawn to indicate the ideal behavior of the system. Agreement of the data with the calculated curves is very good, especially for those corrected with the activity coefficients.

Consequently, the composition of solid condensates is proved to be estimated successfully by the method described above.

\section{5 About the results of Reid}

Reid $^{(\theta)}$ carried out several runs to separate benzene and cyclohexane mixtures at total reflux. Since the system forms simple eutectics, the phase equilibrium can be estimated easily. As he suggested that the runs were made at total vapor pressures of 5.0 to $7.2 \mathrm{mmHg}$, the eutectic point may be at 35 to $36 \%$ of benzene and at -27 to $-31{ }^{\circ} \mathrm{C}$. The mixture of $58 \%$ benzene was fed as a melt and frozen at $-50^{\circ} \mathrm{C}$ or below, while the vapor was kept at 32 to $50^{\circ} \mathrm{C}$ to vaporize the solid. He obtained $99 \%$ benzene at the top and 47 to $54 \%$ at the bottom. If one evaluates the separation in terms of conventional separation efficiency, it can be seen to lie from 0.15 to 0.37 , while for ideal separation it should be 0.61 . Therefore, his assumption that the system completely attains equilibrium could not hold. It can be said that his device could not act as a multistage one as expected. However, from the results of the present study it may well be said that the device could be more effective if the cooling bath is maintained at a little below the eutectic point and the vaporizing section at a little above the equilibrium temperature to avoid total condensation and total vaporization of the vapor and solid respectively.

\section{Conclusion}

A method for estimating the composition of solid condensates on a cold surface from a mixed vapor for a binary system is derived, including an overall coefficient for condensation. To determine the values of the coefficient, experiments of measuring the condensation rate of the individual components were carried out.

As a result, the condensation rate was found to depend on the flow characteristics of the gas, thermal conditions of the system and condenser dimensions. Using these empirical correlations, the composition of condensate was estimated. Good agreement was found between calculated and experimental compositions as a function of the temperature of the cold surface.
Appendix: Derivation of Eqs. (4) and (5)

From Eqs. (1) and (3),

$$
K_{i} C\left(y_{i}-y_{i}^{*}\right) d A=-\bar{u} S C d y_{i}
$$

This can be rewritten with the boundary conditions as

$$
\int_{0}^{A} \frac{K_{i}}{\bar{u} S} d A=\int_{y_{i 2}}^{y_{i 1}} \frac{d y_{i}}{y_{i}-y_{i}{ }^{*}}
$$

where $S=(\pi / 4) D^{2}, d A=\pi D d l$ and $A=\pi D L$.

Then with the definition of $\bar{K}_{i}$ (Eq. (6)), Eq. (A-2) can be integrated assuming that $y_{i}^{*}$ is constant to yield

$$
\frac{4}{\bar{u} D} \int_{0}^{L} K_{i} d l=\frac{4 L \bar{K}_{i}}{\bar{u} D}=\ln \frac{y_{i 1}-y_{i}^{*}}{y_{i 2}-y_{i}{ }^{*}}
$$

Thus one obtains

$$
y_{i 2}-y_{i}{ }^{*}=\left(y_{i 1}-y_{1}^{*}\right) \exp \left(-4 L \bar{K}_{i} / \vec{u} D\right)
$$

While integrating Eq. (3) over the whole length one obtains

$$
\int_{0}^{N_{i}} d N_{i}=N_{i}=\int_{y_{i 1}}^{y_{i 2}}-\bar{u} C S d y_{i}=\bar{u} S C\left(y_{i 1}-y_{i 2}\right)
$$

Substitution of $y_{i 2}$ from Eq. (A-5) in Eq. (A-4) results, after some arrangements, in

$$
N_{i}=\tilde{u} S C\left[1-\exp \left(-4 L \bar{K}_{i} / \bar{u} D\right)\right]\left(y_{i 1}-y_{i}{ }^{*}\right)
$$

By comparison of Eq. (A-6) with Eq. (4), which is the definition of $k_{i}$, Eq. (5) for $k_{i}$ is derived.

\section{Aknowledgment}

The authors wish to express thanks to Mrs. Michiko Abe, Y. Katayama, Y. Tanaka, and A. Yamaguchi for their help in carring out the experiments.

\section{Nomenclature}

$=$ surface area

$C \quad=$ mean molar density of vapor-gas mixture

$D \quad=$ inside diameter of tube $\quad[\mathrm{cm}]$

$\mathscr{D} \quad=$ diffusion coefficient $\left[\mathrm{cm}^{2} / \mathrm{sec}\right]$

$K_{i} \quad=$ local overall coefficient for condensation

$\bar{K}_{i} \quad=$ average overall coefficient for condensation

$[\mathrm{cm} / \mathrm{sec}]$

$k_{i} \quad=$ proportionality constant in Eq. (4) [cm/sec]

$L \quad=$ length of condenser $[\mathrm{cm}]$

$l \quad=$ distance along condenser $\quad[\mathrm{cm}]$

$N_{i} \quad=$ average rate of condensation $\quad[\mathrm{mol} / \mathrm{sec}]$

$P(T) \quad=$ vapor pressure at $T \quad[\mathrm{mmHg}]$

$p \quad=$ partial pressure $\quad[\mathrm{mmHg}]$

$S \quad=$ cross-sectional area of tube $\quad\left[\mathrm{cm}^{2}\right]$

$\begin{array}{lll}T & =\text { absolute temperature } & \\ & & \\ & & \\ \end{array}$

$=$ temperature $\quad\left[{ }^{\circ} \mathrm{C}\right]$

$\bar{u} \quad=$ mean velocity of gas $\quad[\mathrm{cm} / \mathrm{sec}]$

$y=$ vapor composition $[-]$

$Z \quad=$ average composition of condensate $\quad[--]$

$z \quad=$ local composition of condensate [-]

$=$ ratio of $k_{i}$, defined by Eq. (9)

$=$ factor defined by Eq. (11)

$=$ activity coefficient

$=$ viscosity

$=$ total pressure

$=$ density

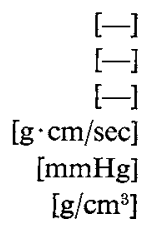

$\langle$ Subscripts and Superscript $\rangle$

$A, B=$ component of binary mixture

$i \quad=$ either $A$ or $B$ 


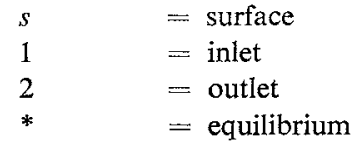

\section{Literature Cited}

1) Davies, M. and J. I. Jones: Trans. Faraday Soc., 50, 1042 (1954).

2) Fowler, L., W. N. Trump and C. F. Vogler: J. Chem. Eng. Data, 13, 209 (1968).

3) Gillot, J. and W. M. Goldberger: Chem. Eng. Progr., Symp. Ser., 65 (91), 36 (1969).

4) Holden, C. A. and H. S. Bryant: Separation Science, 4, 1
(1969).

5) Matsuoka, M. and H. Tanaka: Preprints for 45th Annual Meeting of The Soc. of Chem. Engrs., Japan, G-116, Osaka (1980).

6) Reid, A. F.: Ind. Eng. Chem., 43, 2151 (1951).

7) Vitovec, J., J. Smolik and J. Kugler:Collect. Czech. Chem. Commun., 43, 396 (1978).

8) Walsh, P. N. and N. O. Smith: J. Chem. Eng. Data, 6, 33 (1961).

9) idem: J. Phys. Chem., 65, 718 (1961).

(Presented at the 12th Autumn Meeting of The Soc. of Chem. Engrs., Japan, at Okayama, October 1978.)

\title{
REACTION MECHANISM OF NO- $\mathrm{NH}_{3}-\mathrm{O}_{2}$ SYSTEM, ELUCIDATED BY TRANSIENT RESPONSE TECHNIQUE
}

\author{
HiRoo NIIYAMA, Kimiaki SASAMOTO, SHIzUo YOSHIDA \\ AND ETSURO ECHIGOYA \\ Department of Chemical Engineering, \\ Tokyo Institute of Technology, Tokyo 152
}

\begin{abstract}
Transient response technique was applied to elucidate the roles of oxygen in $\mathrm{NO}-\mathrm{NH}_{3}-\mathrm{O}_{2}$ reaction system at low concentrations of $\mathrm{NO}$ and $\mathrm{NH}_{3}$ (ca. $500 \mathrm{ppm}$ ). Reaction hardly takes place in the absence of oxygen. Analyses of transient response curves of such reaction system indicated that the reaction involves consumption of surface oxygen. High activity of $\mathrm{CuSO}_{4}$ Active Carbon catalyst is due to its high affinity for oxygen.
\end{abstract}

\section{Introduction}

Many studies have been made on the reaction of $\mathrm{NO}$ with $\mathrm{NH}_{3}$. The approaches to the subject may be divided into two groups. One is the "practical approach" in which catalytic activity, life, durability against $\mathrm{SO}_{2}$, etc., are of primary concern ${ }^{2,3,6,14,16)}$. These studies were done in flow reactors and with low reactant concentrations. They are practically important, but it is difficult to obtain microscopic information about the surface process. The other is the "surface chemical approach" in which isotope labeling technique and many instrumental analyses have been successfully applied ${ }^{1,9,10,13,15)}$. They have given much useful informations about the surface processes, but uncertainty remains as to whether they are applicable to practical conditions or not. As Miyamoto et al..$^{8}$ pointed out, the reaction mechanism may be different in the low-concentration range.

The objective of the present study is to elucidate

Received October 31, 1980. Correspondence concerning this article should be addressed to $H$. Nilyama. the reaction mechanisms under conditions close to practical cleaning of exhaust gases. $\mathrm{CuSO}_{4}$ on active carbon, was used as catalyst, which has a high catalytic activity. It is stated ${ }^{4,11,12}$ that sulfate catalysts have a high durability against $\mathrm{SO}_{2}$, although this is questionable in the low-temperature region ${ }^{12}$.

The striking feature of the reaction, $\mathrm{NO}-\mathrm{NH}_{3}$, is the remarkable increase in rate promoted by oxy$\operatorname{gen}^{7,9,10,15)}$ which is comprised in the major advantage for exhaust gas treatment from stationary sources. In the present study emphasis will be placed on the roles of oxygen in the surface process. Transient Response Technique $^{5)}$ (TRT) was applied for this purpose. One of the difficulties in applying the technique to surface catalysis is that such transient behavior can be observed for only a limited time. This is because, in a usual circumstances, the number of active sites or intermediates is small in comparison with the number of gaseous molecules entering into the system. At low concentrations of reactants, longer time will be necessary for the consumption of intermediates and transient behavior will be prolonged 